\title{
Absence of spontaneous magnetization in low-dimensional Anderson and Kondo lattices
}

\author{
C. Proetto $\left({ }^{1}\right)$ and A. Lopez $\left({ }^{2}\right)$ \\ Centro Atomico Bariloche $\left({ }^{3}\right)$ and Instituto Balseiro $\left({ }^{4}\right)$, 8400-Bariloche, Argentina
}

(Reçu le 3 mai 1983, accepté le ler juin 1983)

\begin{abstract}
Résumé. - Utilisant l'inégalité de Bogoliubov, nous prouvons rigoureusement qu'un réseau d'Anderson ou Kondo ne peut pas avoir d'aimantation spontanée à température finie.

Abstract. - It is rigorously proved by use of Bogoliubov's inequality that, at finite temperature, a one or two dimensional Anderson or Kondo lattice can have no spontaneous lattice or sublattice magnetization.
\end{abstract}

The possibility of phase transitions at finite temperature in low-dimensional systems has been discussed at different occasions in the literature by use of the so-called Bogoliubov inequality [1-3]. Mermin and Wagner [3] have proved the absence of spontaneous magnetization for Heisenberg spin systems in low-dimensionality. As these results are not directly applicable to itinerant electron systems, Walker and Rujgbrok [3], and later Ghosh [3], have extended the proof to Hubbard type Hamiltonians.

In this paper we apply Bogoliubov's inequality to the Anderson and Kondo lattices and show the absence of spontaneous magnetization at finite temperature for these cases. Although this could hardly be a surprise, we feel that the large amount of theoretical and experimental work devoted to mixed valence or Kondo systems [4] in the last years calls for exact results in this field.

There is experimental evidence on ordering $[5,6]$ in some Ce compounds, such as $\mathrm{CeAl}_{2}$, $\mathrm{Ce}_{3} \mathrm{Al}_{11}$ and $\mathrm{CeB}_{6}$. From this point of view there seems to be no need to discuss one or two dimensional systems. However most theoretical treatments simplify considerably when applied to low-dimensional models, so that exact results or bounds are a useful test for approximate theories.

The case of the Anderson lattice is interesting because the nature of the exchange interactions it contains is quite different from those considered by Mermin-Wagner for the Heisenberg

( ${ }^{1}$ ) Consejo Nacional de Investigaciones Cientificas y Técnicas, Argentina.

$\left({ }^{2}\right)$ Present address : Centre de Recherches sur les Très Basses Températures, C.N.R.S., 25 avenue des Martyrs, BP 166 X, 38042 Grenoble Cedex, France.

$\left({ }^{3}\right)$ Comision Nacional de Energia Atomica.

$\left({ }^{4}\right)$ Comision Nacional de Energia Atomica and Universidad Nacional de Cuyo. 
spin case. The effective exchange interactions are fourth order in the hybridization matrix element [7] and are mediated by the conduction band. The interactions between localized moments can be of the RKKY type but in general depend on band structure and band filling. They are anisotropic and become antiferromagnetic for a semiconductor or insulator. A canonical transformation shows [7] that at higher orders there are multisite interactions, not included in the arguments of Mermin-Wagner.

It is thus important to find exact bounds to the possibility of magnetic ordering for this model and to see how these bounds depend upon the different parameters of the original Hamiltonian. These results would be complementary to recent calculations of the ground state properties [8].

In this letter we show that the bounds for spontaneous magnetization in the Anderson lattice depend on characteristics of the conduction band only through a second moment of the hopping integral and not of the effective exchange interaction as a crude extension of the Mermin-Wagner results would imply. These bounds are independent of the hybridization parameter and of the different Coulomb-repulsion integrals.

The Kondo lattice, in turn, may be considered either on its own merits or as an approximation to the Anderson lattice for the case of integer valence. Exact results in this case complement approximate calculations [9] which show the existence of an ordered ground state, with antiferromagnetic correlations.

Our proof thus applies to a whole class of many body Hamiltonians, of which those of Hubbard, Anderson lattice, Falikov-Kimball and Kondo lattice are special cases. The common feature shared by these models is the existence of a vector order parameter, which leads to the instability of the assumed ordered ground state for $d<2$ against the creation at finite temperature, of an arbitrary large number of spin wave excitations.

For the Anderson lattice we start from the Hamiltonian

$$
\begin{aligned}
H_{\mathrm{AL}}=\sum_{i j \sigma} t\left(\mathbf{R}_{i}\right. & \left.-\mathbf{R}_{j}\right) c_{i \sigma}^{+} c_{j \sigma}+\sum_{i \sigma} E_{0} f_{i \sigma}^{+} f_{i \sigma}+\frac{U}{2} \sum_{i \sigma} f_{i \sigma}^{+} f_{i \sigma} f_{i \bar{\sigma}}^{+} f_{i \bar{\sigma}}+ \\
& +G \sum_{i}\left(\sum_{\sigma} f_{i \sigma}^{+} f_{i \sigma}\right)\left(\sum_{\sigma^{\prime}} c_{i \sigma^{\prime}}^{+} c_{i \sigma^{\prime}}\right)+\frac{I}{2} \sum_{i \sigma} c_{i \sigma}^{+} c_{i \sigma} c_{i \bar{\sigma}}^{+} c_{i \bar{\sigma}} \\
& +\sum_{i j \sigma}\left[V\left(R_{i}-R_{j}\right) c_{i \sigma}^{+} f_{j \sigma}+\text { h.c. }\right]-H \sum_{i}\left(S_{i z}+s_{i z}\right) \mathrm{e}^{-i q \cdot \mathbf{R}_{i}}
\end{aligned}
$$

where we have used conventional notation [7] and the last term represents a space dependent static magnetic field interacting with localized and itinerant electrons.

We define local spin operators in the form

$$
S_{i+}=f_{i \uparrow}^{+} f_{i \downarrow} ; \quad S_{i-}=f_{i \downarrow}^{+} f_{i \uparrow} ; \quad S_{i z}=\frac{1}{2}\left(f_{i \uparrow}^{+} f_{i \uparrow}-f_{i \downarrow}^{+} f_{i \downarrow}\right)
$$

and conduction electron spin as

$$
s_{i+}=c_{i \uparrow}^{+} c_{i \downarrow} ; s_{i-}=c_{i \downarrow}^{+} c_{i \uparrow} ; s_{i z}=\frac{1}{2}\left(c_{i \uparrow}^{+} c_{i \uparrow}-c_{i \downarrow}^{+} c_{i \downarrow}\right) .
$$

The Fourier transforms are given by

$$
\begin{gathered}
\mathbf{S}(\mathbf{k})=\sum_{i} \mathrm{e}^{i \mathbf{k} \cdot \mathbf{R}_{i}} \mathbf{S}_{i} ; \quad \mathbf{s}(\mathbf{k})=\sum_{i} \mathrm{e}^{i \mathbf{k} \cdot \mathbf{R}_{i}} \mathbf{s}_{i} \\
f_{i \sigma}=\frac{1}{\sqrt{N}} \sum_{\mathbf{k}} \mathrm{e}^{-i \mathbf{k} \cdot \mathbf{R}_{i}} f_{\mathbf{k} \sigma} ; \quad c_{i \sigma}=\frac{1}{\sqrt{N}} \sum_{\mathbf{k}} \mathrm{e}^{-i \mathbf{k} \cdot \mathbf{R}_{i}} c_{\mathbf{k} \sigma}
\end{gathered}
$$

where $N$ is the number of lattice sites.

The quantity $t\left(\mathbf{R}_{i}\right)$ can be taken as real and is even in the argument $\mathbf{R}_{i}$. The properties of $V\left(\mathbf{R}_{i}\right)$, follow from the parity of $d$ and $f$ functions [10]. This precludes on-site hybridization and implies 
that $V\left(\mathbf{R}_{i}\right)$ is odd under the change $\mathbf{R}_{i} \rightarrow-\mathbf{R}_{i}$. However since in many theoretical models it is assumed that $V\left(\mathbf{R}_{i}\right)=0$ except for $\mathbf{R}_{i}=0$, we shall comment on the consequences of this assumption for the bounds that we obtain.

The Bogoliubov inequality that we use reads

$$
\frac{1}{2}\left\langle\left\{A, A^{+}\right\}\right\rangle\left\langle\left[[B, H], B^{+}\right]\right\rangle \geqslant T|\langle[B, A]\rangle|^{2}
$$

where $H$ is the Hamiltonian of the system, $A$ and $B$ are any two bounded operators and $\langle\ldots\rangle$ :s the thermal average with the Hamiltonian $H$. Both quantities $\left\langle\left\{A, A^{+}\right\}\right\rangle$and $\left\langle\left[[B, H], B^{+}\right]\right\rangle$ are real and positive definite.

We choose $A=S_{-}(-\mathbf{p}-\mathbf{q})$ and $B=S_{+}(\mathbf{p})+s_{+}(\mathbf{p})$ and obtain

$$
\begin{aligned}
\left\langle\left[\left[B, H_{\mathrm{AL}}\right], B^{+}\right]\right\rangle & =\sum_{i} t\left(\mathbf{R}_{i}\right) W\left(\mathbf{R}_{i}\right)\left(\mathrm{e}^{i \mathbf{p} . \mathbf{R}_{i}}-1\right)+ \\
& +\sum_{i}\left[V\left(\mathbf{R}_{i}\right) K\left(\mathbf{R}_{i}\right)\left(\mathrm{e}^{i \mathbf{p .} \mathbf{R}_{i}}-1\right)+\text { c.c. }\right]+2 H\left\langle S_{z}(-\mathbf{q})+s_{z}(-\mathbf{q})\right\rangle
\end{aligned}
$$

where

$$
W\left(\mathbf{R}_{i}\right)=\sum_{\mathbf{k} \boldsymbol{\sigma}} \mathrm{e}^{i \mathbf{k} \cdot \mathbf{R}_{i}}\left\langle c_{\mathbf{k} \sigma}^{+} c_{\mathbf{k} \sigma}\right\rangle
$$

and

$$
K\left(\mathbf{R}_{i}\right)=\sum_{\mathbf{k}} \mathrm{e}^{i \mathbf{k} \cdot \mathbf{R}_{i}}\left\langle c_{\mathbf{k} \uparrow}^{+} f_{\mathbf{k} \uparrow}-c_{\mathbf{k} \downarrow}^{+} f_{\mathbf{k} \downarrow}\right\rangle .
$$

Making use of the parity properties

$$
t\left(\mathbf{R}_{i}\right)=t\left(-\mathbf{R}_{i}\right), \quad V\left(\mathbf{R}_{i}\right)=-V\left(-\mathbf{R}_{i}\right), \quad W\left(\mathbf{R}_{i}\right)=W\left(-\mathbf{R}_{i}\right), \quad K\left(\mathbf{R}_{i}\right)=K\left(-\mathbf{R}_{i}\right) .
$$

Equation 6 can be written

$$
\begin{array}{rl}
\left\langle\left[\left[B, H_{\mathrm{AL}}\right], B^{+}\right]\right\rangle=\sum_{i} & t\left(\mathbf{R}_{i}\right) W\left(\mathbf{R}_{i}\right)\left(\cos \mathbf{p} \cdot \mathbf{R}_{i}-1\right) \\
& \quad-2 \operatorname{Im} \sum_{i} V\left(\mathbf{R}_{i}\right) K\left(\mathbf{R}_{i}\right) \sin \left(\mathbf{p} \cdot \mathbf{R}_{i}\right)+2 H\left\langle S_{z}(-\mathbf{q})+s_{z}(-\mathbf{q})\right\rangle \\
\left\langle\left[\left[B, H_{\mathrm{AL}}\right], B^{+}\right]\right\rangle \equiv \alpha(\mathbf{p})+\beta(\mathbf{p})+\gamma \leqslant|\alpha(\mathbf{p})|+\beta(\mathbf{p})+|\gamma|
\end{array}
$$

where $\alpha(\mathbf{p}), \beta(\mathbf{p})$ and $\gamma$ refer to each one of the terms in equation 7 .

The term $|\alpha(\mathbf{p})|$ can be bounded as follows

$$
\begin{aligned}
&|\alpha(\mathbf{p})|=\left|\sum_{i} t\left(\mathbf{R}_{i}\right) W\left(\mathbf{R}_{i}\right)\left(\cos \mathbf{p} \cdot \mathbf{R}_{i}-1\right)\right| \leqslant \\
& \leqslant \sum_{i}\left|t\left(\mathbf{R}_{i}\right)\right|\left|W\left(\mathbf{R}_{i}\right)\right|\left|1-\cos \mathbf{p} \cdot \mathbf{R}_{i}\right| \leqslant \sum_{i}\left|t\left(\mathbf{R}_{i}\right)\right| 2 N \frac{R_{i}^{2} p^{2}}{2} \equiv N p^{2} t^{(2)}
\end{aligned}
$$

$t^{(2)}$ is a second moment of the hopping term and we have used the fact that

$$
\left|1-\cos \left(\mathbf{p} . \mathbf{R}_{i}\right)\right| \leqslant \frac{p^{2} R_{i}^{2}}{2} \text { and }\left|W\left(\mathbf{R}_{i}\right)\right| \leqslant 2 N
$$

since $\left|\left\langle c_{\mathbf{k} \sigma}^{+} c_{\mathbf{k} \sigma}\right\rangle\right| \leqslant 1$.

With these transformations, the inequality equation 5 can be written

$$
\frac{1}{2}\left\langle\left\{S_{-}(-\mathbf{p}-\mathbf{q}), S_{+}(\mathbf{p}+\mathbf{q})\right\}\right\rangle \geqslant \frac{N T\left|S_{0 z}(-\mathbf{q})\right|^{2}}{t^{(2)} p^{2}+\hat{\beta}(\mathbf{p})+|\gamma|}
$$


where we have called

$$
\begin{aligned}
& S_{0 z}(-\mathbf{q})=\frac{1}{N}\left\langle S_{z}(-\mathbf{q})\right\rangle, \quad s_{0 z}(-\mathbf{q})=\frac{1}{N}\left\langle s_{z}(-\mathbf{q})\right\rangle, \\
& |\gamma|=2\left|H\left(S_{0 z}(-\mathbf{q})+s_{0 z}(-\mathbf{q})\right)\right| \text { and } \hat{\beta}(\mathbf{p})=\frac{1}{N} \beta(\mathbf{p}) .
\end{aligned}
$$

Summing this last expression over $\mathbf{p}$, the first term gives

$$
\begin{aligned}
\sum_{\mathbf{p}}\left\langle\left\{A, A^{+}\right\}\right\rangle & =N \sum_{i}\left\langle\left(S_{i-} S_{i+}+S_{i+} S_{i-}\right)\right\rangle \\
& =N \sum_{i}\left\langle\left(c_{i \downarrow}^{+} c_{i \uparrow}-c_{i \downarrow}^{+} c_{i \downarrow}\right)^{2}\right\rangle \leqslant N^{2} .
\end{aligned}
$$

We thus have

$$
\frac{N}{2} \geqslant T\left|S_{0 z}(-\mathbf{q})\right|^{2} \sum_{\mathbf{p}} \frac{1}{t^{(2)} p^{2}+\hat{\beta}(\mathbf{p})+|\gamma|} .
$$

In the limit of large $N$, we can replace the sum by an integral, obtaining

$$
\frac{1}{2} \geqslant T\left|S_{0 z}(-\mathbf{q})\right|^{2}\left(\frac{L^{d}}{N}\right) \frac{\Omega(d)}{(2 \pi)^{d}} \int_{0}^{p_{0}} p^{d-1} \mathrm{~d} p \int \mathrm{d} \mu \frac{1}{t^{(2)} p^{2}+\hat{\beta}(\mathbf{p})+|\gamma|} .
$$

where $\Omega(d)$ is the area of the unit sphere, $\mathrm{d} \mu$ is the normalized angular measure in $d$-dimensions and $p_{0}$ defines the limit of the first Brillouin zone.

The quantity $\hat{\beta}(\mathbf{p})$ is such that its angular average vanishes. If one chooses an on-site hybridization, $\hat{\beta}(\mathbf{p})$ is identically zero, as can be seen from equation 6 . Using Jensen's inequality we can write [12]

$$
\begin{aligned}
\int_{0}^{p_{0}} p^{d-1} \mathrm{~d} p \int \mathrm{d} \mu \frac{1}{\left[t^{(2)} p^{2}+\hat{\beta}(\mathbf{p})+|\gamma|\right]} & \geqslant \int_{0}^{p_{0}} p^{d-1} \mathrm{~d} p \frac{1}{\int \mathrm{d} \mu\left[t^{(2)} p^{2}+\hat{\beta}(\mathbf{p})+|\gamma|\right]} \\
& =\int_{0}^{p_{0}} p^{d-1} \mathrm{~d} p \frac{1}{t^{(2)} p^{2}+|\gamma|} .
\end{aligned}
$$

We thus obtain for :

one dimension

$$
\begin{aligned}
\left|S_{0 z}(-\mathbf{q})\right|^{2} & \leqslant \frac{\pi}{2 T \rho}\left[2 t^{(2)}\left|H\left(S_{0 z}(-\mathbf{q})+s_{0 z}(-\mathbf{q})\right)\right|\right]^{1 / 2} \\
& \times\left\{\operatorname{arctg}\left[\left(\frac{t^{(2)}}{\left|2 H\left(S_{0 z}(-\mathbf{q})+s_{0 z}(-\mathbf{q})\right)\right|}\right)^{1 / 2} p_{0}\right]\right\}^{-1}
\end{aligned}
$$

where $\rho=N / L^{d}$ is the density. For sufficiently small $H$ we can write [11]

$$
\frac{\left|S_{0 z}(-\mathbf{q})\right|^{2}}{\left|S_{0 z}(-\mathbf{q})+s_{0 z}(-\mathbf{q})\right|^{1 / 2}} \leqslant \frac{\sqrt{2 t^{(2)}}}{T \rho}|H|^{1 / 2}
$$

proving the vanishing of $\left|S_{0 z}(-\mathbf{q})\right|^{2}$ as $H \rightarrow 0$; 


\section{two dimensions}

$$
\left|S_{0 z}(-\mathbf{q})\right|^{2} \leqslant \frac{2 \pi t^{(2)}}{T \rho}\left\{\ln \left[1+\frac{t^{(2)} p_{0}^{2}}{2\left|H\left(S_{0 z}(-\mathbf{q})+s_{0 z}(-\mathbf{q})\right)\right|}\right]\right\}^{-1} \ldots
$$

For small $H$ this can be written

$$
\left|S_{0 z}(-\mathbf{q})\right|^{2} \leqslant \frac{2 \pi t^{(2)}}{T \rho}\left|\ln \left(\frac{2 \mid H\left(S_{0 z}(-\mathbf{q})+s_{0 z}(-\mathbf{q}) \mid\right.}{t^{(2)} p_{0}^{2}}\right)\right|^{-1}
$$

leading again to the vanishing of $\left|S_{0 z}(-q)\right|^{2}$ as $H \rightarrow 0$;

three dimensions

$$
\begin{aligned}
\left|S_{0 z}(-\mathbf{q})\right|^{2} & \leqslant \frac{\pi^{2} t^{(2)}}{T \rho}\left[p_{0}-\left(\frac{2\left|H\left(S_{0 z}(-\mathbf{q})+s_{0 z}(-\mathbf{q})\right)\right|}{t^{(2)}}\right)^{1 / 2}\right. \\
& \left.\times \operatorname{arctg}\left[p_{0}\left(\frac{t^{(2)}}{2\left|H\left(S_{0 z}(-\mathbf{q})+s_{0 z}(-\mathbf{q})\right)\right|}\right)^{1 / 2}\right]\right]^{-1} .
\end{aligned}
$$

For small $H$ we find that $\left|S_{0 z}(-q)\right|^{2}$ is bounded by a finite quantity so that our argument is inconclusive.

The Kondo lattice Hamiltonian can be derived from (1) by means of a second order canonical transformation $[7,9]$. The relevant terms obtained give

where

$$
H_{\mathbf{K L}}=\sum_{i j \sigma} t\left(\mathbf{R}_{i}-\mathbf{R}_{j}\right) c_{i \sigma}^{+} c_{j \sigma}+\sum_{i j l} J_{i j l} \mathbf{S}_{i} \cdot \mathbf{s}_{j l}-H \sum_{i}\left(S_{i z}+s_{i z}\right) \mathrm{e}^{-i \mathbf{q} \cdot \mathbf{R}_{i}}
$$

with

$$
J_{i j l}=\frac{1}{2 N^{2}} \sum_{\mathbf{k} \mathbf{k}^{\prime}} V_{\mathbf{k}} V_{\mathbf{k}^{\prime}}^{*} \mathrm{e}^{i \mathbf{k} \cdot \mathbf{R}_{i j}} \mathrm{e}^{i \mathbf{k}^{\prime} \cdot \mathbf{R}_{l i}}\left[D\left(\varepsilon_{k}\right)+D\left(\varepsilon_{\boldsymbol{k}^{\prime}}\right)-D\left(\varepsilon_{\mathbf{k}}-U\right)-D\left(\varepsilon_{\mathbf{k}^{\prime}}-U\right)\right]
$$

$$
\begin{aligned}
& D(\varepsilon)=\left(\varepsilon-E_{0}\right)^{-1}, \quad \mathbf{R}_{i j}=\mathbf{R}_{i}-\mathbf{R}_{j} ; \quad V_{\mathbf{k}}=\sum_{i} V\left(\mathbf{R}_{i}\right) \mathrm{e}^{i \mathbf{k} \cdot \mathbf{R}_{i}} \\
& s_{j l+}=c_{j \uparrow}^{+} c_{l \downarrow} ; \quad s_{j l-}=c_{j \downarrow}^{+} c_{l \uparrow} ; \quad s_{j l z}=\frac{1}{2}\left(c_{j \uparrow}^{+} c_{l \downarrow}-c_{j \downarrow}^{+} c_{l \downarrow}\right) .
\end{aligned}
$$

By suitable choice of $J_{i j l}$ we can obtain from equation 20 several cases dealt with in the literature. The case where $J_{i j l}=J_{0} \delta_{i j} \delta_{i l}$ is the one most often considered and it has been discussed in one dimension in reference $9 a$, using a real space renormalization group technique. In the work of reference $9 b$ the authors include also the case where $J_{i j l}=J_{1} \delta_{i j}$.

Taking $A$ and $B$ as above, the commutator $\left[\left[B, H_{\mathrm{KL}}\right], B^{+}\right]$has a contribution from the hopping term in (20) which is identical to the one previously calculated and another contribution from the exchange part. The latter gives

$$
\begin{aligned}
\left\langle\left[\left[B, \bar{H}_{\mathrm{ex}}\right], B^{+}\right]\right\rangle= & \sum_{i j l} J_{i j l}\left\{\left[F_{j l}(\mathbf{p})-2 F_{j i}(\mathbf{p})-2 F_{i l}(\overline{\mathbf{p}})\right]\left\langle S_{i z} s_{j l z}\right\rangle\right. \\
& \left.-\frac{1}{2}\left[F_{i j}(\mathbf{p})+F_{i l}(\overline{\mathbf{p}})\right]\left\langle S_{i+} s_{j l-}+S_{i-} s_{j l+}\right\rangle\right\}
\end{aligned}
$$

where $F_{i j}(\mathbf{p})=1-\cos \left(\mathbf{p} \cdot \mathbf{R}_{i j}\right)$. We have made use of the fact that

and

$$
J_{i j l} \equiv J\left(\mathbf{R}_{i}, R_{j}, R_{l}\right)=J\left(-\mathbf{R}_{i},-\mathbf{R}_{j},-\mathbf{R}_{l}\right)
$$

$$
\left\langle S_{i z} s_{j, l+}\right\rangle=\left\langle S_{-i z} s_{-j,-l+}\right\rangle \text {, etc. }
$$


Equation 22 can be bounded as follows

$$
\left|\left\langle\left|\left[\left[B, H_{\mathrm{ex}}\right], B^{+}\right]\right|\right\rangle\right| \leqslant \frac{1}{2} \sum_{i j l}\left|J_{i j l}\right|\left[\frac{1}{2} F_{j l}(\mathbf{p})+2 F_{i j}(\mathbf{p})+2 F_{i l}(\mathbf{p})\right]
$$

where we have used $\left|\left\langle S_{i z} s_{j l z}\right\rangle\right| \leqslant\left\|S_{i z} s_{j l z}\right\| \leqslant\left\|S_{i z}\right\| \cdot\left\|s_{j l z}\right\| \leqslant \frac{1}{4},\|A\|$ being the norm of the operator $A$.

The right hand side of equation 23 is smaller than

$$
p^{2} \frac{1}{2} \sum_{i j l}\left|J_{i j l}\right|\left[R_{i j}^{2}+R_{i l}^{2}+\frac{1}{4} R_{j l}^{2}\right] \equiv N p^{2} J^{(2)}
$$

where $J^{(2)}$ is a second moment of $\left|J_{i j l}\right|$. Using this result we finally get

$$
\frac{1}{2} \geqslant T\left|S_{0 z}(-\mathbf{q})\right|^{2}\left(\frac{L^{d}}{N}\right) \frac{\Omega(d)}{(2 \pi)^{d}} \iint \frac{p^{d-1} \mathrm{~d} p \mathrm{~d} \mu}{p^{2}\left[t^{(2)}+J^{(2)}\right]+|\gamma|} .
$$

The integral over $\mathrm{d} \mu$ in equation 25 can be readily done and the integral over $p$ leads to an expression similar to (14), with $t^{(2)}+J^{(2)}$ replacing $t^{(2)}$. Thus the conclusions are exactly the same as for the Anderson lattice, namely that there is no spontaneous magnetic ordering at finite temperature in one and two dimensions.

\section{Acknowledgments.}

We thank Prof. E. H. Lieb for pointing out the use of Jensen's inequality and for fruitful discussions. Conversations with B. Alascio and C. Balseiro were very helpful.

\section{References}

[1] LiEB, E. H. and Wu, F. Y., Phys. Rev. Lett. 20 (1968) 1445.

[2] Hohenberg, P. C., Phys. Rev. 158 (1967) 383.

[3] a) Mermin, N. D. and Wagner, H., Phys. Rev. Lett. 17 (1966) 1133.

b) Mermin, N. D., Phys. Rev. 176 (1968) 250.

c) Walker, M. B. and RujugbroK, Th. W., Phys. Rev. 171 (1968) 513.

d) GHosh, D. K., Phys. Rev. Lett. 27 (1971) 1584.

[4] See for instance the Proceedings of the Conference in Valence Instabilities, edited by P. Wachter and H. Boppart (North-Holland, Amsterdam) 1982.

[5] a) Barbara, B., Boucherle, J. X., Buevoz, J. L., Rossignol, M. F. and Schweizer, J., J. Physique Colloq. 40 (1979) C5-321.

b) Chouteau, G. and Palleau, G., J. Magn. Magn. Mat. 15-18 (1980) 311.

[6] Rossat-Mignod, J., Burlet, P., Kasuya, T., Junit, S. and Komatsubara, T., Solid State Commun. 39 (1981) 471.

[7] Proetto, C. and Lopez, A., Phys. Rev. B 24 (1981) 3031.

Proetto, C. and Lopez, A., Phys. Rev. B 25 (1982) 7037.

[8] Jullien, R. and Martin, R. M., J. Appl. Phys. 53 (1982) 2137.

Varma, C. M., SchlÜter, M. and YafeT, Y., in Valence Instabilities, edited by P. Wachter and H. Boppart (North-Holland) 1982.

Schlottmann, P. and Gonçalves Da Silva, C. E. T., in Valence Instabilities, edited by P. Wachter and H. Boppart (North-Holland) 1982.

[9] a) Jullien, R., Pfeuty, P., Bhattacharjee, A. K. and Coqblin, B., J. Appl. Phys. 50 (1979) 7555.

b) Lopes, L. C., Jullien, R., Bhattacharjee, A. K. and Copblin, B., Phys. Rev. B 26 (1982) 2640.

[10] Balseiro, C., Passeggi, M. and Alascio, B., Solid State Commun. 16 (1975) 737.

Stevens, K. W. H., J. Phys. C. 11 (1978) 985.

[11] In reference 3.c) $|\operatorname{arctg} x|$ is approximated by $|x|$, which would give a wrong bound in equation 15 , since $\operatorname{arctg}$ is in the denominator. We have taken instead the limiting value for $H \rightarrow 0$, i.e. $|\operatorname{arctg} x| \rightarrow \pi / 2$.

[12] Reed, M. and Simon, B., Methods of Modern Mathematical Physics (Academic Press, New York) 1979. 